\title{
OPTICAL BEAM DIAGNOSTICS FOR THE LNLS SYNCHROTRON LIGHT SOURCE ${ }^{*}$
}

\author{
R.H.A. Farias, L.C. Jahnel, Liu Lin and P.F. Tavares \\ Laboratório Nacional de Luz Síncrotron - Caixa Postal 6192 - 13083-970 Campinas SP - Brazil
}

\begin{abstract}
An optical characterization bench for the electron beam in the LNLS storage ring using visible synchrotron radiation is described. Preliminary measurements at injection energy $(120 \mathrm{MeV})$ and at operation energy $(1.37 \mathrm{GeV})$ are presented. The system includes a high frame rate (up to $400 \mathrm{~Hz}$ ) CCD camera and a fast photodiode (7 ps FWHM) for longitudinal measurements.
\end{abstract}

\section{INTRODUCTION}

The Brazilian Synchrotron Light Source (LNLS) is based on a $1.37 \mathrm{GeV}$ electron storage ring with a $120 \mathrm{MeV}$ injector Linac. Commissioning of the storage ring [1] at low energy started on May 1996 and presently, an year later, we can store $120 \mathrm{~mA}$ at $120 \mathrm{MeV}$ and ramp more than $75 \mathrm{~mA}$ to $1.37 \mathrm{GeV}$. The availability of a real time image of the beam in the control room proved to be very useful both during commissioning and normal operation of the ring. In addition, measurements of the stored electron beam transverse and longitudinal profiles yield information about the beam emittance and bunch length. Both quantities are affected to first order by lattice functions, and to higher order by current dependent collective effects. The ability to measure these quantities accurately is, thus, a very important tool to asses both the lattice focusing properties and the effects of collective phenomena, such as ion trapping and beam instabilities.

Several means of producing an image are described in the literature, including pinholes to collimate X-rays $[2,3]$ or lenses to focus the visible part of the spectrum $[4,5,6]$. The LNLS UVX storage ring operating at injection energy $(120 \mathrm{MeV})$ does not produce detectable $\mathrm{X}$-ray intensities and the present beam characterization bench is set for visible light observation. The achieved resolution of about $70 \mu \mathrm{m}$, limited by diffraction due to the natural radiation opening angle, is well suited for the injection energy, where the large beam size is dominated by intra-beam scattering effects. At high energy, however, we expect difficulties to measure the small vertical beam size as well as the vertical emittance, since the latter is calculated from the beam size and the knowledge of the beta functions. In our case, the vertical emittance resolution is about $0.26 \mathrm{~nm}$.rad.

\section{THE OPTICAL BEAM DIAGNOSTICS BEAMLINE}

The UVX optical electron beam characterization bench uses visible radiation produced at dipole ADI01 at a 4 degree low dispersion port. A schematic layout is shown in Figure 1. The high energy synchrotron radiation power is absorbed by a sapphire filter attached to a water cooled copper radiation mask. The visible light is then extracted into air through a sapphire vacuum window and guided by two mirrors ( 86 and 90 degree deflection) to the experimental hall. Two borosilicate plano-convex lenses form an image of the electron beam on the surface of a CCD sensor. A variable aperture is placed in front of the radiation exit port to define the horizontal-vertical optical acceptance of the line and consequently control diffraction and geometrical curvature effects. The visible radiation is monochromatized by a $10 \mathrm{~nm}$ bandpass interference filter to reduce chromatic aberrations. A set of two polarization filters is used to polarize and control beam intensity on the CCD sensor. The filters are placed near the detector to minimize potential errors due to their surface irregularities. The first mirror, located behind the shielding wall, is mounted on a platform which allows remote angular adjustments about the horizontal and vertical axes. The polarization filters can be commanded from the control room in order to adjust the beam intensity level at the CCD.

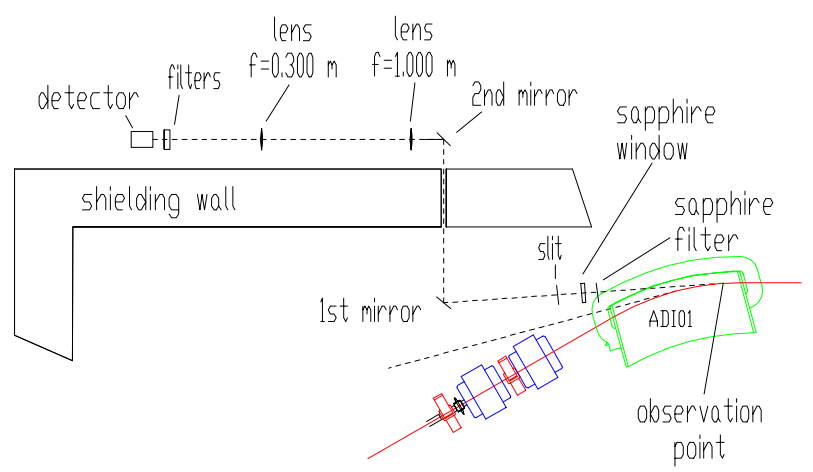

Figure 1: The LNLS UVX optical characterization bench.

The main line CCD camera (EEVCAM17-46), with $512 \times 512$ square pixels $15 \times 15 \mu \mathrm{m}$ in size, will be able to operate up to $400 \mathrm{~Hz}$ frame rate, allowing measurements of phenomena which occur in time scales as short as $10 \mathrm{~ms}$ such as beam damping at high energy. Meantime,

\footnotetext{
* This work is partially supported by 'Fundação de Amparo à Pesquisa do Estado de São Paulo'.
} 
while the EEV camera is not operational, we have installed a common CCD video camera to perform the preliminary measurements.

A broadband dielectric beamsplitter divides a fraction of the light to a second branch of the characterization bench where a calibrated photodiode is used to monitor beam current and beam current decay variations for lifetime measurement purposes.

A second beamsplitter separates a fraction of light from the second branch to a third one. Here an ultrafast commercial photodetector (Picometrix PX-D7) with 7 ps FWHM response time will be used to measure single shot bunch length.

\subsection{Optical resolution}

The synchrotron light accepted by the aperture of the optical bench comes from an arc along the electron orbit. The longer the arc the greater the apparent transverse and longitudinal sizes of the source. If the aperture is made smaller, the limit to resolution from diffraction becomes worst. The optimum aperture is thus a compromise between these effects. In the case of visible synchrotron radiation, the natural diffraction limit imposed by the angular width of the emitted radiation is usually dominant. The optimum aperture should, thus, be approximately equal to the natural radiation opening angle as the diffraction limit will not improve by increasing the aperture but the apparent source size will. For UVX running at $1.37 \mathrm{GeV}$, the width of the radiation cone for $550 \mathrm{~nm}$ is $\pm 3.6 \mathrm{mrad}$. The calculated resolution of $70 \mu \mathrm{m}$ is dominated by diffraction, as expected. A bandpass filter with $10 \mathrm{~nm}$ FWHM is used to eliminate the long wavelength light which would degrade the diffraction limited resolution. The calculated parameters for UVX standard operation mode are given in Table I. The large value for the natural emittance at $120 \mathrm{MeV}$ is due to intra-beam scattering effects. The transverse spatial resolution of $70 \mu \mathrm{m}$ corresponds to emittance resolutions of $0.26 \mathrm{~nm} . \mathrm{rad}$ and $3.5 \mathrm{~nm}$.rad for the vertical and horizontal planes respectively. The principal limitations are expected in the vertical plane at $1.37 \mathrm{GeV}$ whenever coupling coefficients are less than approximately $0.3 \%$.

Table I: Parameters at the UVX beam diagnostics line.

\begin{tabular}{|l|c|c|}
\hline Injection energy & 120 & $\mathrm{MeV}$ \\
\hline Operation energy & 1.37 & $\mathrm{GeV}$ \\
\hline Horizontal beta & 1.4 & $\mathrm{~m}$ \\
\hline Vertical beta & 18.9 & $\mathrm{~m}$ \\
\hline Natural emittance & & \\
\hline at $1.37 \mathrm{GeV}$ & 99.8 & nm.rad \\
\hline at $120 \mathrm{MeV}$ & $\approx 900$ & nm.rad \\
\hline
\end{tabular}

\section{EXPERIMENTAL RESULTS}

\subsection{Emittance Measurements}

A conventional $\mathrm{CCD}$ video camera has been installed at the main beam line to perform static beam emittance measurements. The beam image is captured using a frame grabber and the beam sizes are calculated by fitting gaussian functions to the horizontal and vertical beam profiles.

Beam transverse profiles have been measured as a function of current for the injection and operation energies. Figure 2 shows the beam image seen at the monitor in the control room.
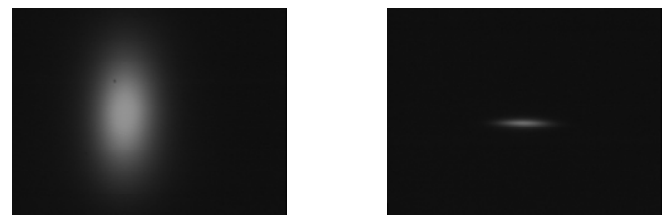

Figure 2: Beam images from dipole ADI01 4 degree exit port corresponding to $\mathrm{E}=120 \mathrm{MeV}$ (left) and $\mathrm{E}=1.37 \mathrm{GeV}$ (right).

Figure 3 shows the fitted gaussian curves for an horizontal and a vertical beam profile. Figures $4 \mathrm{a}$ and $4 \mathrm{~b}$ show the beam sizes (one standard deviation) measured as a function of beam current for $1.37 \mathrm{GeV}$ and $120 \mathrm{MeV}$. Figures $5 \mathrm{a}$ and $5 \mathrm{~b}$ show the natural emittance and coupling factor for both energies. The vertical emittance and coupling at $1.37 \mathrm{GeV}$ are lower than $0.3 \mathrm{~nm} . \mathrm{rad}$ and $0.3 \%$, respectively, and are limited by the measurement resolution. The measured natural emittance is in good agreement with the theoretical value at $1.37 \mathrm{GeV}$ indicating that the linear optics of the machine is close to the calculated one. The slight increase in vertical beam size for low currents is probably due to a slow drift in the electron beam orbit which defocus the image on the CCD sensor, since the automatic orbit correction system was not activated during the measurements. At $120 \mathrm{MeV}$, the measured emittance at low currents is close to the value predicted from intra-beam scattering calculations. For higher currents we have measured an enormous increase in beam emittance. This is probably due to the fact that high current measurements need to be performed right after injection, when the beam is not yet damped (the damping time is $10 \mathrm{~s}$ ).
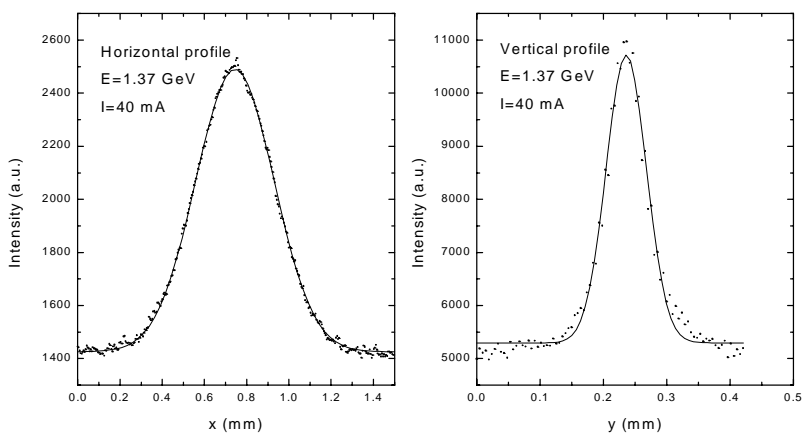

Figure 3: Horizontal (left) and vertical (right) beam profiles for $\mathrm{E}=1.37 \mathrm{GeV}$ and $\mathrm{I}=40 \mathrm{~mA}$. The solid curve is a gaussian fitted to the experimental data (dots). 


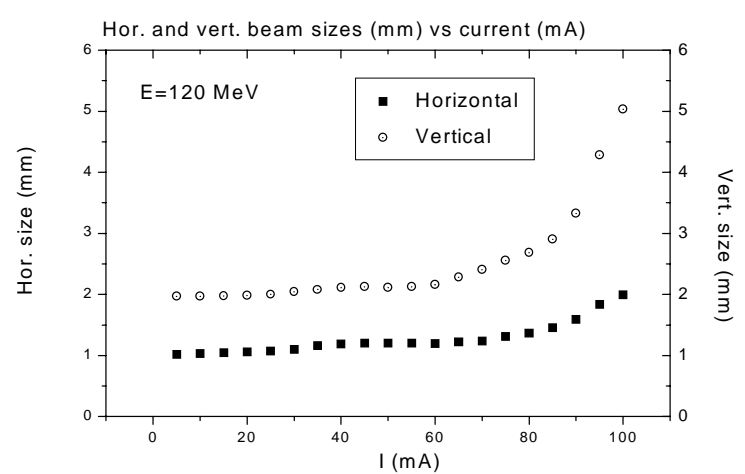

Figure 4a: Horizontal and vertical r.m.s. beam size as a function of beam current for $\mathrm{E}=120 \mathrm{MeV}$.

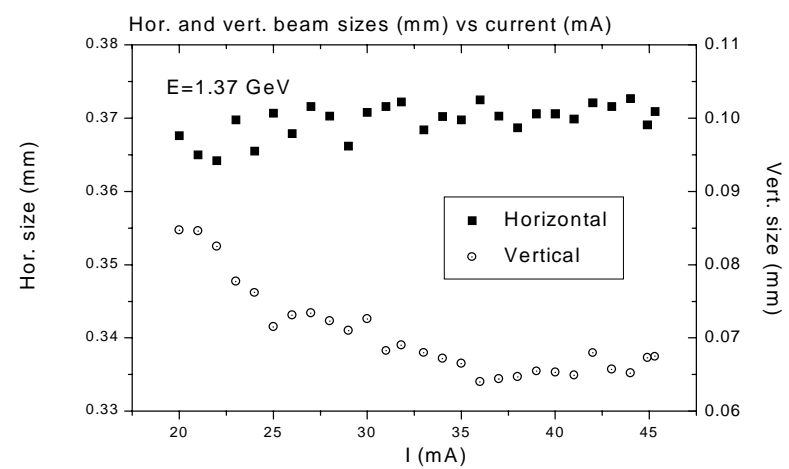

Figure 4b: Horizontal and vertical r.m.s. beam size as a function of beam current for $\mathrm{E}=1.37 \mathrm{GeV}$.

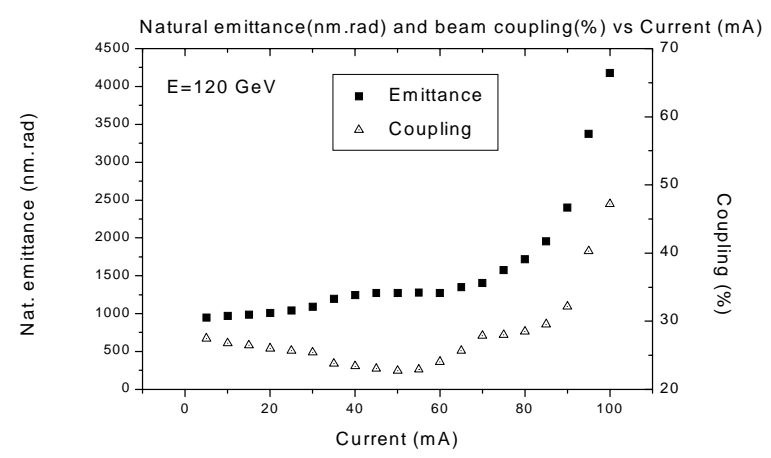

Figure 5a: Natural emittance and coupling as a function of beam current for $\mathrm{E}=120 \mathrm{MeV}$.

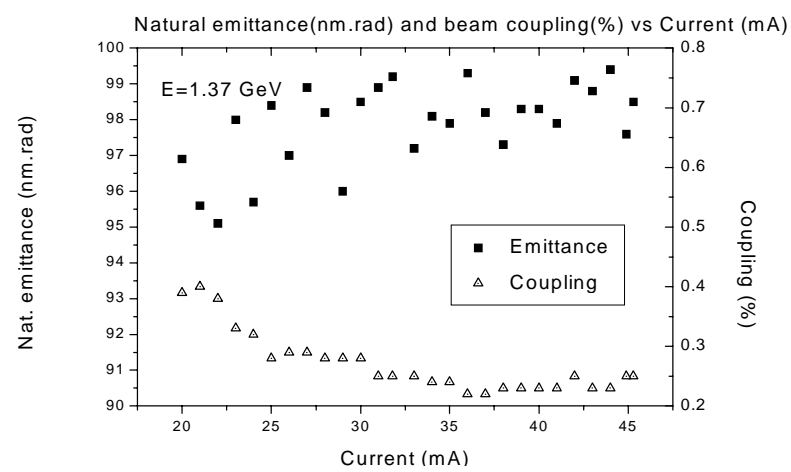

Figure 5b: Natural emittance and coupling as a function of beam current for $\mathrm{E}=1.37 \mathrm{GeV}$.

\subsection{Observation of ion trapping}

Effects of ion trapping have been observed in the storage ring at the synchrotron light monitor in the control room. Ion effects have been detected as a vertical expansion of the beam when a kicker is excited. The vertical enlargement of the beam persists even when the excitation is turned off. The beam can be made flat again by applying appropriate voltages to the clearing electrodes. Figure 6 shows two images of the beam at 900 $\mathrm{MeV}$ corresponding to the cases before and after a kicker excitation.
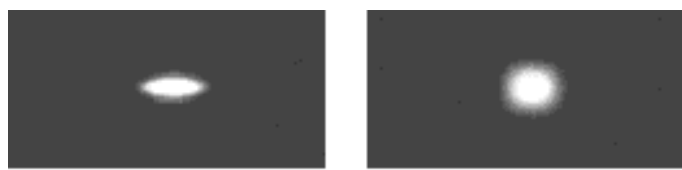

Figure 6: Images showing a vertical enlargement of the beam after excitation with a kicker.

\section{CONCLUSIONS}

The LNLS optical beam diagnostic line using visible synchrotron radiation has been described. Although not fully operational, some transverse beam size and emittance measurements have been carried out. The installation of the fast photodetector and the high frame rate CCD camera will allow measurements in the longitudinal plane as well as analysis of damping in the transverse plane.

The emittance measurements show that the system resolution is adequate for analysis at injection energy but is limited for measurements in the vertical plane at nominal operation energy. The results show, however, a vertical emittance lower than $0.3 \mathrm{~nm}$.rad at $1.37 \mathrm{GeV}$, or coupling coefficient lower than $0.3 \%$, indicating a careful alignment of the magnets.

Some improvements in the beamline are in progress including a system for remote focusing of the beam image and modification of the beam extraction scheme. The new scheme will use a refrigerated mirror in the vacuum chamber in place of the sapphire filter, which has become darkened by radiation.

The limitation to resolution comes from inherent diffraction effects, so resolution improvement imply decreasing the wavelength towards X-rays. The idea of a new line using X-ray imaging is also being considered.

\section{REFERENCES}

[1] A.R.D.Rodrigues et al, 'Commissioning and Operation of the Brazilian Synchrotron Light Source', these proceedings.

[2] C.Bovet and E.Rosa, Workshop on Advanced Beam Instrumentation, KEK, 1991.

[3] E.Tarazona and P.Elleaume, Rev Sci.Inst. 66, Feb 95.

[4] A.Hofmann, IEEE Tran.Nuc.Sci., NS-28,No.3, 1981.

[5] Åke Andersson, Juri Tagger, Nuclear Instruments and Methods, A 364 (1995) 4-12.

[6] R.J.Nawrocky, J.Galayda, L.H.Yu and D.M.Shu, IEEE Trans. Nuc. Sci., NS-32, No. 5, 1985. 